\title{
Heliospheric Magnetic Field Configuration at Solar Maximum Conditions: Consequences for Galactic Cosmic Rays
}

\author{
T. H. Zurbuchen*, R. von Steiger ${ }^{\dagger}$, W. B. Manchester*, and L.A. Fisk* \\ *Department of Atmospheric, Oceanic, and Space Sciences, University of Michigan, \\ Ann Arbor, MI 48109-2143, USA \\ ${ }^{\dagger}$ International Space Science Institute, Hallerstrasse 6, CH-3012 Bern, Switzerland
}

\begin{abstract}
During solar maximum conditions, the heliosphere is highly structured on all spatial scales. It is the purpose of this paper to summarize our current understanding of these structures from global scales to mesoscale, a fraction of $1 \mathrm{AU}$. We use theoretical considerations, in situ observations near Earth and the Ulysses spacecraft, and global heliosphere calculations to discuss the effects on both global and mesoscales on the three-dimensional structure of the heliospheric magnetic field and their effects on galactic cosmic rays. These conclusions are in contrast to nearsolar-minimum-like heliospheric conditions that are currently assumed in modulation and transport calculations even during solar maximum. The expected complex heliospheric properties should be of major importance for the interpretation of the heliospheric boundary events observed by Voyager 1 since 2002. A companion paper by L. A. Fisk will explore the effects of the mesoscale structures on particle acceleration in the heliospheric boundary region.
\end{abstract}

\section{INTRODUCTION}

Since completion of the Voyagers' planetary missions in 1989 with the close flyby of Neptune by the Voyager 2 spacecraft, the Voyagers have embarked on a new quest, racing to the boundary of the heliosphere. The existence of this boundary has been predicted based on the pressure balance of the solar wind and the interstellar pressure, constrained by in situ observations and the anticipated properties of the galactic environment. In 2002, after traversing the heliosphere for close to two solar cycles, Voyager 1 has reached this boundary region [1], and possibly passed through the solar wind termination shock into the subsonic region [2].

During the time period of interest, the outer heliosphere is dominated by solar wind associated with solar maximum conditions. Figure 1a shows, as a function of time, the particle observations from Voyager's LEPC instrument [3], combined with solar and heliospheric measures of solar activity. These measures have been time-shifted to Voyager 1 assuming a constant average solar wind speed. More rigorous propagation methods have been performed by Wang [4] and Wang and Richardson [5]. These calculations model stream interactions in the solar wind and also account for the slowdown of the solar wind due to mass-loading by interstellar pickup ions. During solar maximum conditions, these computations result in an average speed of $410 \mathrm{~km} / \mathrm{s}$

\footnotetext{
CP719, Physics of the Outer Heliosphere: Third International IGPP Conference, edited by V. Florinski, N. V. Pogorelov, and G. P. Zank

(C) 2004 American Institute of Physics 0-7354-0199-3/04/\$22.00
} 


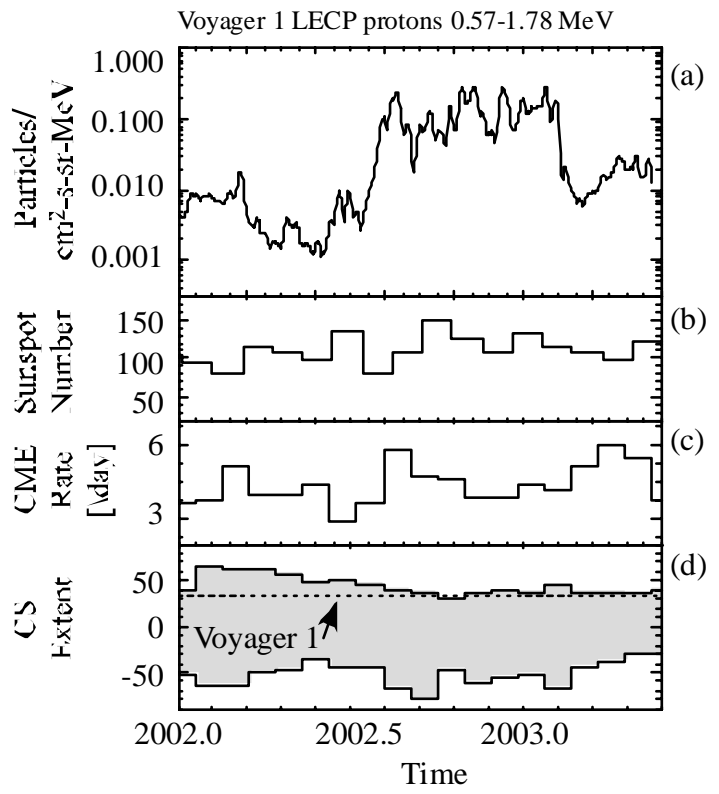

FIGURE 1. Voyager 1 heliospheric boundary events in context with solar and solar wind conditions. (a) Voyager 1 LECP proton fluxes in the energy range of $0.57-1.78 \mathrm{MeV}$ [2]. Solar and heliospheric conditions propagated to Voyager 1 using a constant propagation speed of $410 \mathrm{~km} / \mathrm{s}$. (b) Sunspot number (from NOAA). (c) CME rate per month (from [6]). (d) Current sheet latitude range computed from solar magnetic field (from [7]), with over-plotted Voyager 1 trajectory.

used for Fig. 1 [personal communication, J. Richardson]. Figure 1b shows the sunspot number provided by the National Oceanic and Atmospheric Administration (http://www.ngdc.noaa.gov/stp/ SOLAR/solar.html), time-shifted using this constant speed. Figure 1c shows the CME number per day observed by SOHO [6] using the same computations. Figure 1d shows the latitudinal extent of the heliospheric current sheet calculated by Riley et al. [7] at 30 solar radii. The heliographic latitude of the Voyager 1 spacecraft is over-plotted as a dashed line. This model would therefore predict that, during the heliospheric boundary events, Voyager is generally above the current sheet - it barely enters into the region of maximum extent. The evolution of the current sheet in the deep heliosphere should therefore be very important for the accurate prediction of the Voyager 1 space environment. Such detailed three-dimensional calculations of the outer heliosphere currently do not exist.

Figure 1 provides a survey of the solar and heliospheric context governing the Voyager 1 heliospheric boundary events. The sunspot number is close to its maximum value during this solar cycle, but with significant variability from month to month. The elevated activity and rapidly changing photospheric magnetic field is the cause for coronal mass ejections (CMEs) that appear at rates up around 5 per day. These CMEs preferentially originate close to the current sheet, with significantly smaller rates at latitudes $>60 \mathrm{deg}[8,6]$. This can be understood in the context of the structure of the 
global heliosphere, which is calculated by Riley et al. based on observations of the global solar magnetic field. These observations are the basis of global magnetohydrodynamics (MHD) calculations under the assumption of time-stationary conditions during every Carrington rotation. This may not be a good assumption close to solar activity maximum, as indicated by the increased number of CMEs in Fig. 1. These CMEs can lead to abrupt changes in the solar and heliospheric magnetic field configuration [8]. It may therefore be surprising that the MHD model compares favorably with Ulysses and ACE observations in the inner heliosphere [7]. This may indicate that, in most cases, CMEs contribute to a gradual change of the heliospheric magnetic field configuration, as opposed to a single abrupt global change, as is sometimes suggested.

There are important limitations in our current understanding of the three-dimensional structure and topology of the heliospheric magnetic field. All calculations assume either time-stationary conditions or model time-evolution only in the context of single events, such as CMEs. However, there is mounting experimental and theoretical evidence for the effects of time-dependent evolution even in the absence of CMEs. One important example is so-called interchange reconnection in the corona, which likely has a dominant role in the evolution of the corona and the heliospheric magnetic field $[9,10$, 11]. These reconnections lead to time-dependent motions in the solar corona described by a velocity field $\vec{u}_{\perp}(t)$ on the source surface. Under some general assumptions (for details, see [12]), the resulting magnetic field perturbations can be calculated using equation (1).

$$
\vec{B}_{\perp}=-\vec{u}_{\perp}\left(\frac{r}{r_{s}}\right) \frac{B_{r}}{V} .
$$

Here, $B_{r}$ is the radial magnetic field, which is $\propto r^{-2} ; V$ is the solar wind speed; and $r_{s}$ is the solar radius. The motion of the solar rotation, $\vec{u}_{\perp}=\Omega r_{s} \sin \theta \hat{e}_{\varphi}$, is the dominant effect under almost all circumstances. However, there are situations when flows from interchange reconnection may dominate [13]. Most systematic solar magnetic field perturbations, when projected into the heliosphere, may be dwarfed by turbulent fluctuations. This makes their direct in situ detection very difficult [14]. However, they likely dominate the magnetic field topology in the heliosphere. This can be on global scales (e.g., [15], [9]) or a mesoscale, for example, in the context of CMEs [10].

There is one additional CME interaction that contributes to systematic mesoscale magnetic field structure at solar maximum. This structure is qualitatively different from the interactions described in equation (1). They arise from non-radial, CME-induced perturbations of the solar wind flow in the inner heliosphere [16, 17]. Figure 2 shows an example of a CME simulation described in detail by Manchester et al. [17].

In the absence of efficient heliospheric reconnection, these perturbations are expected to persist throughout the heliospheric transit, expanding in latitude, with latitudinal $\propto r$, with little expansion in radial direction.

The next section will discuss evidence for these mesoscale fluctuations from Ulysses data during the same time period. Solar wind composition data are used in conjunction 


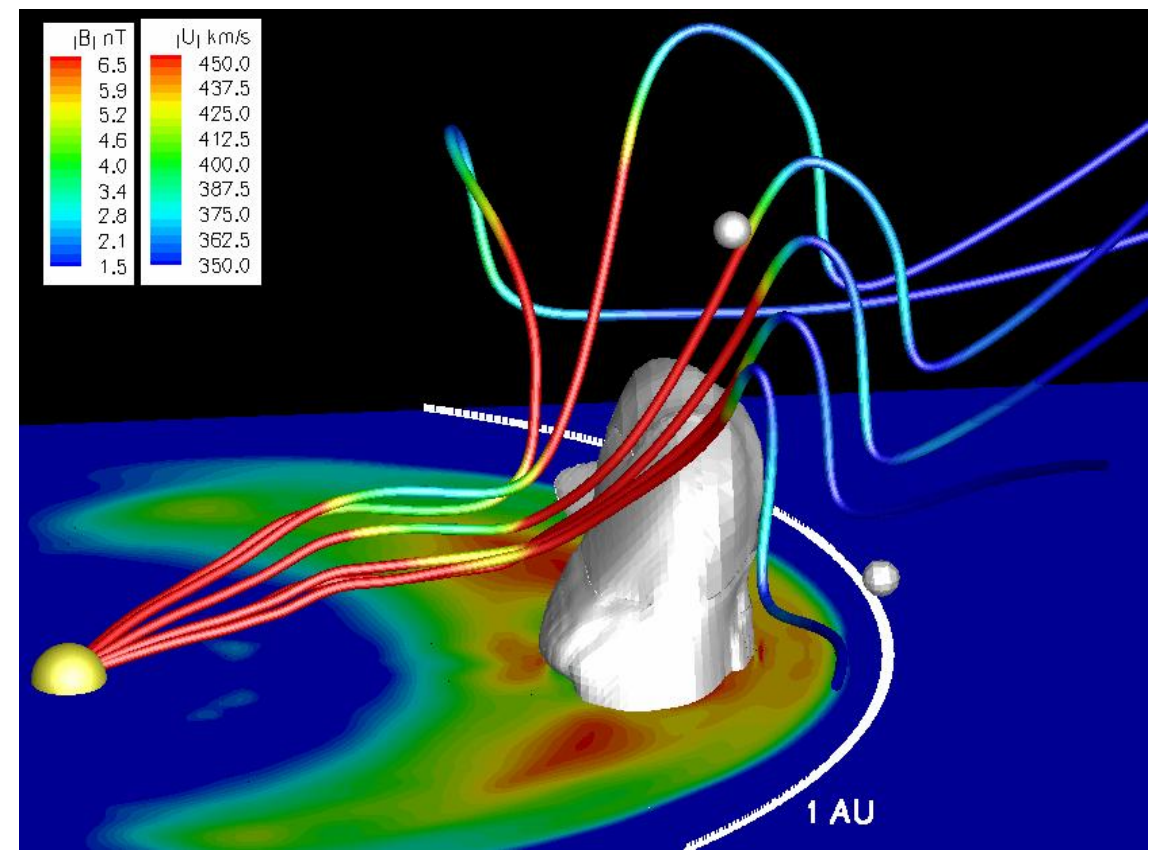

FIGURE 2. Magnetic field perturbations induced by a CME ejection close to an equatorial streamer. The shaded volume represents the coronal ejecta interacting with its local heliospheric environment as an isosurface at $25 \mathrm{nT}$; the color scale in the ecliptic indicates values of solar wind speed, the color scale on magnetic field lines indicates values of magnetic field strength. The resulting magnetic field distortions propagate to high latitudes, far beyond the latitude range of the ejecta.

with plasma and field data to discuss the solar wind. The next two sections deal with theoretical discussions of the overall magnetic field configuration's effect on the structure of the outer heliosphere and its modulation. First, we discuss the effects of large-scale deviations of the heliospheric magnetic field and the drift motions that should be expected to result. Second, we discuss heliospheric consequences of mesoscale fluctuations and their relation to heliospheric turbulence models commonly used.

\section{GLOBAL STRUCTURE OF CORONA AND HELIOSPHERE FROM ULYSSES OBSERVATIONS}

The variability of the heliospheric structure can be seen in every observation available from Ulysses. Figure 3 shows Ulysses data from early June to mid-July 2001. During this time period, Ulysses moved from 1.35 to $1.44 \mathrm{AU}$, and from $14.7^{\circ}$ to $40.7^{\circ}$ during its fast latitude pass. This is a very suitable interval to get a feeling for the solar wind structure. 


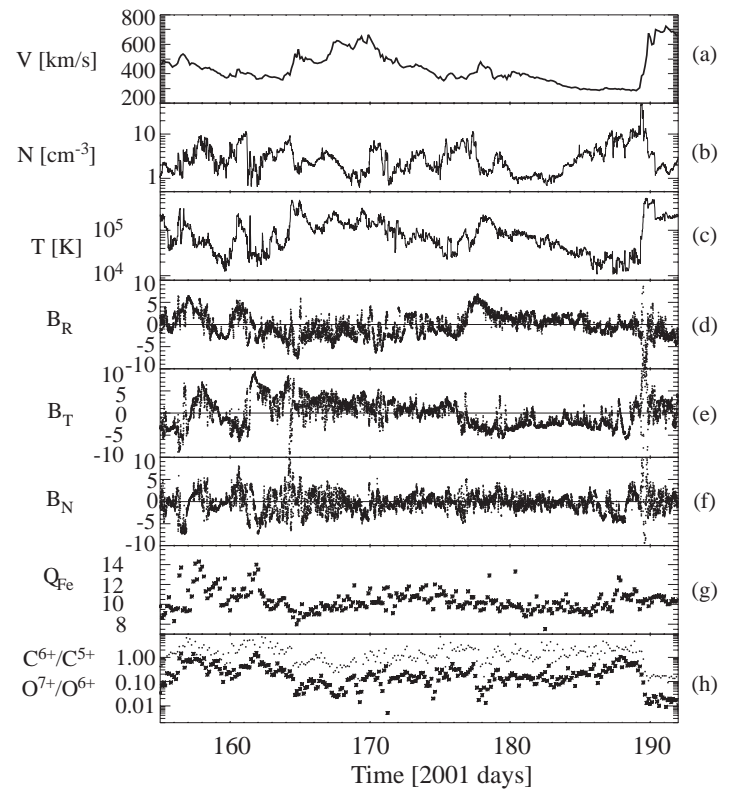

FIGURE 3. Ulysses data from a 35-day interval during the fast latitude scan, when Ulysses crossed Voyager latitudes at approximately the time adequate to understand the heliospheric boundary events shown in Figure 1. The figure shows solar wind speed, density, temperature, three magnetic field coordinates, and charge compositional signatures suitable to identify "hot" CMEs and "cold" coronalhole-associated streams. For details refer to text.

Figure 3 shows plasma, field and ionic composition data, all from their sensors on Ulysses. Figures 1a-c show the solar wind speed, density, and temperature; Figs. 1d-f show the three magnetic field components, and Figs. 1g-h show the ionic composition of the most abundant heavy ions, $\mathrm{Fe}, \mathrm{O}$, and $\mathrm{C}$. It has been pointed out that a combination of these signatures can be used to identify the solar wind regime and distinguish coronalhole-associated wind from fast ICMEs $[18,14]$. Using these signatures, we identify an ICME with very high charge state for all compositional signatures from day 155 to approximately day 164, indicative of at least two ICMEs interacting in the heliosphere. The Fe composition is highly variable, but reaches values in excess of an average charge state of 14 , which is indicative of coronal-source temperatures over $3 \mathrm{MK}$. This ICME exhibits a very strong magnetic field, even though we do not see any smooth rotations indicative of textbook magnetic clouds. Similarly, the temperature decreases to roughly $10 \%$ of the value around the ICME.

This ICME is followed by a stream which is, as seen by the compositional and density signatures, very likely a conglomerate of at least two different streams that interact, causing systematic deflections of the magnetic field in latitude, as seen by brief excursions of $B_{N}$. The decreasing $\mathrm{O}^{7+} / \mathrm{O}^{6+}$ reaches a value below 0.1 during part of this stream, indicative of an edge of fast, coronal-hole-associated stream. The polarity of the magnetic field remains the same $\left(B_{T}>0, B_{R}<0\right)$ during this time period until close to day 177 , when Ulysses crosses the current sheet. Immediately after the crossing, there is 
another signature of coronal-hole solar wind, indicated by $\mathrm{O}^{7+} / \mathrm{O}^{6+}<0.1$. The time Ulysses spends in this stream is very short, but nonetheless visible in compositional and plasma signatures. It is also obvious, based on density, temperature, and field signatures, that this plasma has undergone tremendous interactions, causing compressions and deflections. During days 180-188, Ulysses remains in the same magnetic sector, until it crosses the current sheet again, this time entering into a very fast coronal-hole-associated wind, as indicated by the compositional signatures. Again, the interactions of this stream cause large deflections of the magnetic fields which likely survive their transit to large heliocentric distances. It is important to notice that the stream structure, indicated by compositional signatures and, most importantly, interaction features in $\mathrm{N}$ and $\mathrm{T}$, is very complex, and on a timescale well below the typical spatial scale during solar minimum. The Carrington rotation shown in Fig. 3 is typical for solar wind from all latitudes, containing CME-associated plasma, small streams, and interaction features. This dynamic relaxation does lead to a smoothing and merging of these streams, as seen by the velocity signature. However, in the absence of dominant reconnection, their magnetic field signatures persist. It is the purpose of the subsequent sections to address these structures and their effect on energetic particle transport.

\section{SYSTEMATIC MOTIONS AT LARGE SCALES}

This section neglects mesoscale fluctuations in the field, which will be discussed below, and in a companion paper by Fisk in this volume. It is the purpose of this section to explore the heliospheric magnetic field configuration under the assumptions made by Fisk and Schwadron in 2001 [9]. They showed that, based on the apparent existence of a single current sheet during the entire solar cycle (e.g. [19]), and under the assumption of dominant interchange reconnection, the solar maximum corona is dominated by a rotating current sheet, facilitating the change of polarity at a given pole from one solar minimum to the next. The subsequent re-adjustment of the low- $\beta$ corona will then cause an overall rotation that may be very sporadic, leading to a velocity field $\vec{u}_{C \perp}=\Omega_{C} \hat{e}_{\varphi^{\prime}}$, where $\Omega_{C}(t)$ is the time-dependent rotation of the current sheet, and $\hat{e}_{\varphi^{\prime}}$ is a unit vector perpendicular to the spin axis in the solar equatorial plane. This motion, together with the solar rotation, $\vec{u}_{\perp}=\Omega r_{s} \sin \theta \hat{e}_{\varphi}$, can be included in equation (1) and projected into the heliosphere, under the assumption of a radial solar wind velocity $\mathrm{V}$. The resulting magnetic field configuration can be calculated as described by Zurbuchen et al. [15], under the assumption that $\beta=\pi / 2$, for the equations published there.

$$
\vec{B}=\left(\begin{array}{c}
B_{r} \\
B_{\theta} \\
B_{\varphi}
\end{array}\right)=\left(\begin{array}{c}
B_{0}\left(\frac{r_{0}}{r}\right)^{2} \\
B_{0} \frac{r_{0}^{2}}{V r} \Omega_{C} \sin \left(\varphi+\Omega r / V-\varphi_{0}\right) \\
B_{0} \frac{r_{0}^{2}}{V r}\left[\Omega_{C} \cos \theta \cos \left(\varphi+\Omega r / V-\varphi_{0}\right)-\Omega \sin \theta\right]
\end{array}\right) .
$$


The current sheet rotation, $\Omega_{C}$, results in systematic latitude and longitude transport of field lines, as shown in Fig. 4, under the assumption of constant $\Omega_{C}=\Omega / 8$. It is obvious from this figure that there are significant deviations from Parker configuration, even with the relatively small rotation speed assumed here. As mentioned above, the heliospheric magnetic field lines would have large random and mesoscale systematic variations superposed on this average motion.

There are important consequences to this average motion on gradient and curvature drifts affecting the cosmic ray modulation. Heliospheric drifts are important during solar activity minimum [20], but are not generally considered important during times of solar activity maximum. Solar minimum drifts at high latitude and heliocentric distances large compared to $1 \mathrm{AU}$ tend to be aligned with the polar axis. Parker's magnetic field over the solar poles is, under idealized circumstances, a straight line with direct access to the inner heliosphere. Jokipii et al. [21] have pointed out that the inclusion of random transverse fields would substantially affect the effects of these drifts and enhance particle diffusion.

We now consider the drifts for the field configuration described by equation (2). First, it is useful to determine the geometric location of radial magnetic fields in this
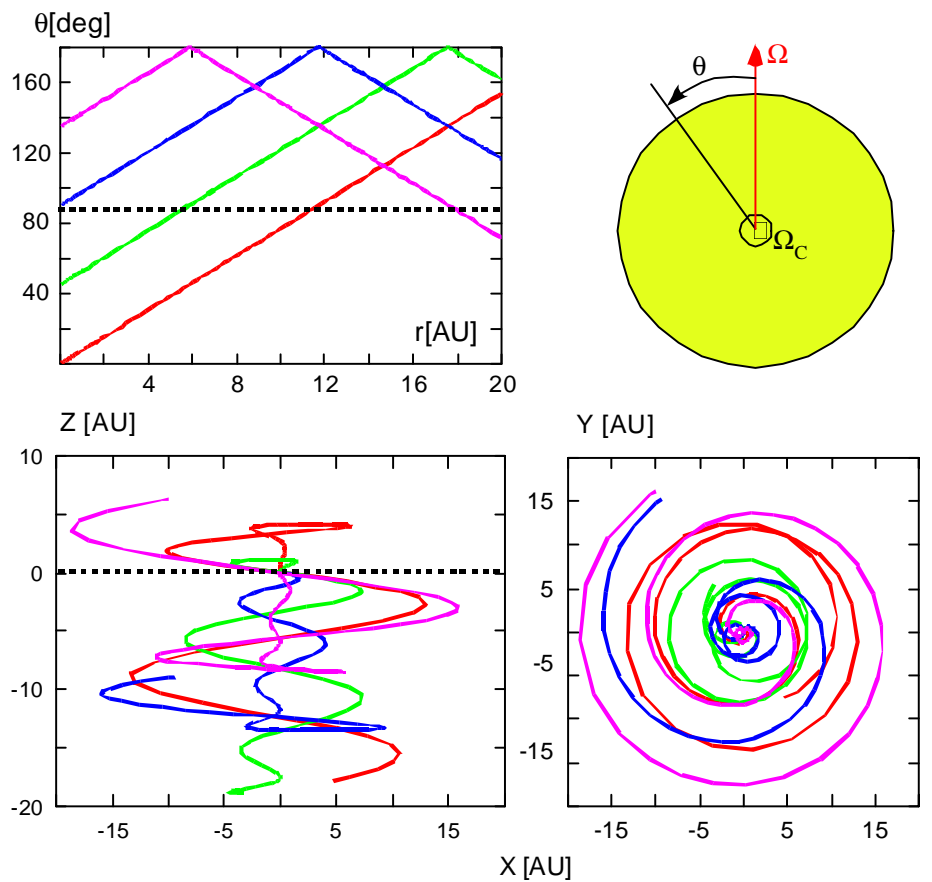

FIGURE 4. Heliospheric magnetic field lines described by equation (8). Field lines are calculated under the assumption that $\Omega_{C}=\Omega / 8$. The top-right plot explains the rotation geometry in a corotating frame. Field lines show substantial latitudinal transport, as seen in the top-left figure. The bottom plots show two-dimensional projections of the field lines: $\mathrm{x}$ and $\mathrm{y}$ are in the solar equatorial plane, $\mathrm{z}$ is perpendicular to that plane. 
configuration, by setting the $\varphi$ and $\theta$ components of the magnetic field to zero. This results in equations that describe the latitude and longitude dependence of that location.

$$
\begin{aligned}
& \varphi-\varphi_{0}=\frac{\Omega r}{V} \quad \text { and } \\
& \sin \theta_{0}=-\frac{\Omega_{C}}{\Omega} .
\end{aligned}
$$

It is obvious from equation (3) that this geometrical location is a Parker spiral. Indeed, drift terms can be integrated using the well-known relation for the drift velocity (e.g., [22]).

$$
\vec{V}_{D} \propto \nabla \times\left(\frac{\vec{B}}{B^{2}}\right)
$$

Figure 5 shows the latitude-radius relation of such drift lines integrated from the inner to the outer heliosphere, compared with Parker field lines, with $\Omega_{C}=0$. The drift lines depicted in Fig. 5 tend to approach a constant latitude with increasing $r$. A detailed analysis of the analytic form of equation (4), and comparisons of analytical integrations, reveal that the asymptotic latitude is precisely $\theta_{0}$, as defined in equation (3).

The magnetic field configuration described in equation (2) provides a simple and useful tool to discuss the effects of drift during times of high solar activity when neglecting mesoscale fluctuations in the field. For the discussion of Fig. 5, consider first that the solar wind current sheet is undergoing a very irregular rotation in latitude, measured by rotation angle $\theta_{C S}$. From one solar minimum to the next, $\theta_{C S}$ changes from $0^{\circ}$ to $180^{\circ}$. Close to $\theta_{C S}=0^{\circ}$ and $\theta_{C S}=180^{\circ}$, solar minimum modulation applies. In

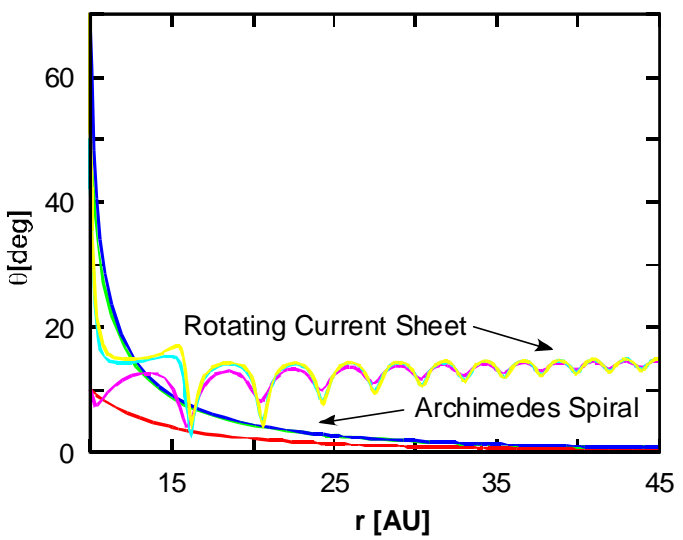

FIGURE 5. Heliocentric drift lines integrated from the inner heliosphere for the field configuration given in Fig. 4 and a standard Archimedes configuration. Different lines indicate different initial latitudes in the heliosphere. Drift equations tend to converge onto a cone with aperture angle $\theta_{0}$ defined in equation (3). 
quasi-stationary conditions, even with $\theta_{C S} \sim 45^{\circ}$, drift terms still play a dominant role, particularly during $q A>0$ cycles, when drifts transport particles through polar regions into the inner heliosphere. Drifts are still effective as long as

$$
\theta_{0}<\left|\theta_{C S}-\pi / 2\right|
$$

If that is no longer the case, drifts would have to penetrate the current sheet, and particle drift orbits extend on both sides of the current sheet. The drifts are therefore effectively shut off.

This is in qualitative agreement with Cummings and Stone [23] who state that, during many years of increasing solar activity level, the particle modulation observed by Voyager 2 does not seem to change substantially. During times of fast rotations of the current sheet close to $\theta_{C S} \sim 60^{\circ}$, increased modulation suddenly sets in, in qualitative agreement with the predictions from this simple model. It should also be noted that coronal mass ejections are often accompanied by abrupt changes in $\theta_{C S}$. These ejections develop in a global merged interaction region, when observed by Voyager, and locally modulate galactic cosmic rays, as observed by Burlaga et al. [24]. However, we argue that it is the global change of the heliospheric magnetic field, described by equation (2), that is causing the increase of global modulation in the heliosphere at solar maximum.

\section{SYSTEMATIC MESOSCALE STRUCTURES}

We have discussed how mesoscale structures make an important contribution to the overall spatial scales governing the solar maximum heliosphere. These motions are systematic in nature and prevalent at all heliospheric latitudes. Some important consequences of this will be discussed by Fisk in a companion paper. We focus here on one specific aspect of these fluctuations, namely their effects on drifts in the heliosphere. It has been shown that those mesoscale fluctuations have important effects on the drifts in the heliosphere [25]. Assuming that mesoscale variations $\delta \vec{B}$ are perpendicular to the background magnetic field, $\vec{B}_{0}$, the ensemble average of the differential streaming through drifts, $\langle\vec{S}\rangle$, can be calculated without assuming that $|\delta \vec{B}|<<\left|\vec{B}_{0}\right|$.

$$
\langle\vec{S}\rangle \propto\left\langle\frac{\vec{B}_{0}}{B_{0}^{2}+(\delta B)^{2}} \times\left(\hat{I}+\frac{\delta \vec{B} \delta \vec{B}}{B_{0}^{2}}\right) \cdot \nabla f_{0}\right\rangle .
$$

Here, $\hat{I}$ is the unity tensor, and $f_{0}$ is the mean distribution function. There are two effects in equation (6) that are important to discuss here. First, mesoscale variations tend to decrease the differential streaming due to drifts. Second, the direction of that streaming can substantially change, if $|\delta \vec{B}| \sim\left|\vec{B}_{0}\right|$. This has important consequences for drifts throughout the heliosphere, as discussed by Fisk and Schwadron [25].

It is important to relate the mesoscale structures to the spatial scale dominated by the evolution of heliospheric turbulence. This is shown in Fig. 6 using a recent model of 


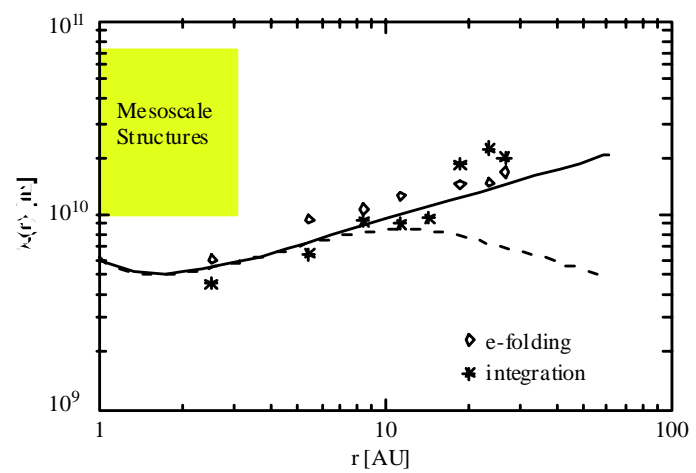

FIGURE 6. Comparison of mesoscale structures with turbulent scales. This figure is adapted from Matthaeus et al. [26]. It compares the correlation scale in a turbulence model with mesoscale structure sizes at $1 \mathrm{AU}$.

heliospheric turbulence for the outer heliosphere. This model calculates the evolution of solar wind turbulence under the influence of solar wind expansion and dynamic effects from pickup ions. At heliocentric distances around $1 \mathrm{AU}$, mesoscale structures are a fraction of $1 \mathrm{AU}$. During their evolution in the heliosphere, they become important as energy-carrying scales that feed the heliospheric turbulence in the inertial range.

\section{SUMMARY}

There are two important aspects to this paper. We first put the heliospheric boundary events observed by Voyager in context with solar activity indicators at the Sun and the heliosphere. We notice that the common approximation of a titled dipole is not sufficient to characterize the heliospheric field and plasma structure. This is mainly caused by a dominance of a new mesoscale at all heliographic latitudes. We also note in passing that we would not expect a dominance of sheared magnetic fields at Voyager latitudes, as predicted by Schwadron and McComas [27]. However, due to the relatively small differences in solar wind speed observed throughout the heliosphere, magnetic field stretching should be less dominant in this case.

Furthermore, the observations at solar maximum conditions predict an important new spatial scale that is intermediate to global scale and turbulence scales. We find that this scale has important effects on gradient and curvature drifts of galactic cosmic ray modulation. These mesoscale structures should be expected to evolve rather independently from the small-scale structure. Important consequences of this are discussed by Fisk [28]. 


\section{ACKNOWLEDGMENTS}

This work was supported, in part, by University of Maryland contracts Z678101 and Q223201, and by JPL contract 1237843. The authors also thank Gary Zank and his group for hosting this meeting on the outer heliosphere.

\section{REFERENCES}

1. F. B. McDonald, E. C. Stone, A. C. Cummings, B. Heikkila, N. Lal and W. R. Webber, Nature 426, 48-51 (2003).

2. S. M. Krimigis et al., Nature 426, 45-48 (2003).

3. S. M. Krimigis et al., Space Sci. Rev. 21, 329-354 (1977).

4. C. Wang, this issue (2004).

5. C. Wang and J. D. Richardson, J. Geophys. Res. 108 (A2), 1058, doi:10.1029/2002JA009322 (2003).

6. N. Gopalswamy, A. Lara, S. Yahiro, S. Nunes and R. A. Howard, "Coronal Mass Ejection Activity During Solar Cycle 23" in Solar Variability as an Input to the Earth's Environment, edited by A. Wilson, ESA SP-535, ESA Publications Division, Noordwijk, 2003, pp. 403-414.

7. P. Riley, J. A. Linker and Z. Mikic, J. Geophys. Res. 107 (A7), 1136, doi:10.1029/2001JA000299 (2002).

8. A. J. Hundhausen, J. Geophys. Res. 98, 13,177-13,200 (1993).

9. L. A. Fisk and N. A. Schwadron, Astrophys. J. 560, 425-438 (2001).

10. A. Reinard, Doctoral Thesis, University of Michigan (2002).

11.N. U. Crooker, J. T. Gosling and S. W. Kahler, J. Geophys. Res. 107, 1028, doi: 10.1029/2001JA000236 (2002).

12.J. V. Hollweg and M. A. Lee, Geophys. Res. Lett. 16, 919-922 (1989).

13. N. Murphy, E. J. Smith and N. A. Schwadron, Geophys. Res. Lett. 29 (22), 2066, doi:10.1029/2002GL51564 (2002).

14.T. H. Zurbuchen, "Heliospheric Magnetic Field Configuration and its Coronal Causes" in Recent Insights into the Physics of the Sun and the Heliosphere, edited by P. Brekke, B. Fleck and J. B. Gurman, Proceedings of IAU Symposium 203, ASP, 2001, p. 585.

15.T. H. Zurbuchen, N. A. Schwadron and L. A. Fisk, J. Geophys. Res. 102 (A11), 24,175-24,182 (1997).

16.D. Odstrcil and V. J. Pizzo, J. Geophys. Res. 104 (A12), 28,225-28,239 (1999).

17.W. B. M. Manchester IV, T. Gombosi, I. Roussev et al., J. Geophys. Res, 109, A01102, doi:10.1029/2002JA009672 (2004).

18.T. H. Zurbuchen, L. A. Fisk, S. T. Lepri and R. von Steiger, "The Composition of Interplanetary Coronal Mass Ejections" in Solar Wind Ten, edited by M. Velli, R. Bruno and F. Malara, AIP Conference Proceedings 679, Melville, New York, 2003, pp. 604-607.

19.E. J. Smith et al., Science 302 (5648), 1165-1169 (2003).

20. J. R. Jokipii, E. H. Levy and W. B. Hubbard, Astrophys. J. 213, 861-868 (1977).

21.J. R. Jokipii, J. Kota, J. Giacalone, T. S. Horbury and E. J. Smith, Geophys. Res. Lett. 22, 3385-3388 (1995).

22.L. A. Fisk, K.-P. Wenzel, et al., Space Sci. Rev. 83, 179-214 (1998).

23.A. C. Cummings and E. C. Stone, American Geophysical Union Spring Meeting 2001 abstract \#SH22F-08 (2001).

24.L. F. Burlaga, N. F. Ness, F. B. McDonald, J. D. Richardson and C. Wang, Astrophys. J. 582, 540-549 (2003).

25.L. A. Fisk and N. A. Schwadron, J. Geophys. Res. 100, 7865-7871 (1995).

26.W. H. Matthaeus, G. P. Zank, C. W. Smith and S. Oughton, Phys. Rev. Lett. 82, 3444-3447 (1999).

27.N. A. Schwadron and D. J. McComas, Geophys. Res. Lett. 30, 1587, doi:10.1029/2002GL016499 (2003).

28.L. A. Fisk, this issue. 